\section{The efficacy of colchicine and dapsone combination therapy in relapsed immune thrombocytopenia}

\author{
Thanawat Rattanathammethee, \\ Wasan Theerajangkhaphichai, \\ Ekarat Rattarittamrong, Sasinee \\ Hantrakool, Chatree Chai-Adisaksopha, \\ Lalita Norasetthada, \\ Adisak Tantiworawit \\ Division of Hematology, Department \\ of Internal Medicine, Faculty of \\ Medicine, Chiang Mai University, \\ Chiang Mai, Thailand
}

\begin{abstract}
The aim of the present paper is to evaluate the efficacy and safety of colchicine and dapsone combination therapy in cases of steroid-dependent, relapsed and refractory immune thrombocytopenia (ITP). This is a retrospective study of ITP patients who attended the Hematology Clinic at Chiang Mai University Hospital (Thailand) from 1 January 2008 to 30 September 2014. Medical records and clinical data were reviewed for efficacy and adverse effects. Sixty-four ITP patients received the combination therapy. The median age was 46 years and $70.3 \%$ were female. The majority $(65.6 \%)$ were relapsed ITP patients. Median platelet count before starting treatment was $22.6 \times 10^{9} / \mathrm{L}$. The response rate was $82.8 \%$, with $75.0 \%$ of patients having a complete response. Median time to response was 8 weeks. The response rate was higher in relapsed patients $(90.4 \%)$ compared to refractory $(61.5 \%)$ and steroid-dependent patients $(77.8 \%)$. Steroid treatment was discontinued in 30 patients $(50 \%)$ following combination therapy. The most common side effect was hemolysis due to dapsone which was found in eight patients $(12.5 \%)$ We can therefore conclude that combination therapy with colchicine and dapsone is an alternative second-line therapy option in relapsed ITP cases with acceptable side effects.
\end{abstract}

\section{Introduction}

Immune thrombocytopenia (ITP) is an acquired immune mediated condition which results in the destruction of platelets and consequently increases the risk of bleeding. ${ }^{1,2}$ The annual incidence of adult
ITP is about 3 per 100,000 persons with the frequency in females being twice that of males. ${ }^{3,4}$ The criteria for the diagnosis of ITP are isolated thrombocytopenia with a platelet count less than $100 \times 10^{9} / \mathrm{L}$ without other explained causes of thrombocytopenia. ${ }^{1}$

A platelet count of less than $30 \times 10^{9} / \mathrm{L}$, as well as thrombocytopenic bleeding or undergoing any invasive procedure are the indications for starting treatment. 2,5 Standard initial therapy is corticosteroids (e.g. prednisolone $1 \mathrm{mg} / \mathrm{kg} / \mathrm{day})^{2,5}$ which confer a $70-80 \%$ response rate within $2-4$ weeks. ${ }^{1,2,6,7}$

Most ITP patients respond to steroid treatment but in some cases, including relapsed, refractory, or steroid-dependent patients, second-line therapy is required. ${ }^{2}$ Second-line treatment aims to prevent prolonged exposure to high dose corticosteroid which is associated with a broad range of adverse or severe side effects.

There are many second-line treatments available for these cases. The second-line therapy for the treatment of ITP includes splenectomy; immunosuppressive drugs such as cyclophosphamide, azathioprine, cyclosporine; anti-CD20 monoclonal antibodies: rituximab; thrombopoietin receptor agonists- eltrombopag and romiplostim; vincristine; danazol; dapsone; and colchicine. The response rate, adverse effects and cost of treatment are varied. ${ }^{2,5}$ Unfortunately, there have been no prospective randomized trials comparing the efficacy and safety of second-line therapy regimens. Data from case series suggest that splenectomy is the most effective secondline therapy for ITP since it has a high response rate and provides a durable response. ${ }^{2,8}$ However, a splenectomy is an invasive procedure which is associated with complications or mortality. ${ }^{8}$ As a result, medical treatment is still widely used in clinical practice.

Dapsone is one of the steroid-sparing agents which can be used in the second-line treatment of ITP. Its mechanism of action is inducing non immune-mediated destruction of red blood cells instead of platelets, resulted in increasing platelet count. ${ }^{5}$ Side effects of dapsone are mild and manageable, e.g., abdominal discomfort, nausea and lowgrade hemolysis. The response rate was 40 $61.8 \%$ and a median duration to response was 3 to 4 months. ${ }^{9-12}$

Colchicine, which prevents platelet destruction by inhibiting the function of microtubules in macrophages, is also considered a second-line therapy for ITP patients. ${ }^{13}$ Dose-dependent diarrhea, nausea and vomiting, are well-known side effects of this medication. ${ }^{13}$ A small study of
Correspondence: Ekarat Rattarittamrong, Division of Hematology, Department of Internal Medicine, Faculty of Medicine, Chiang Mai University, 110 Intravaroros road, A. Muang, Chiang Mai 50200, Thailand. Tel.: +66.53935482 - Fax: +66.53935481, E-mail: ekarat_r@hotmail.com, ekarat.r@cmu.ac.th

Key words: Relapsed immune thrombocytopenia, Colchicine, Dapsone.

Acknowledgement: This study was supported by a research grant from the Faculty of Medicine, Chiang Mai University.

Contributions: TR wrote and revised the paper; WT collected, summarized clinical data, and wrote the paper; ER wrote, analyzed data, and revised the paper; $\mathrm{SH}, \mathrm{CC}, \mathrm{LN}$ revised the manuscript; AT designed the research, obtained researched grant, and wrote the paper.

Conflict of interests: the authors declare no potential conflict of interest.

Received for publication: 9 January 2017 Revision received: 26 January 2017.

Accepted for publication: 5 February 2017.

This work is licensed under a Creative Commons Attribution-NonCommercial 4.0 International License (CC BY-NC 4.0).

C Copyright T. Rattanathammethee et al., 2017 Licensee PAGEPress, Italy

Hematology Reports 2017; 9:7034

doi:10.4081/hr.2017.7034

patients with refractory ITP revealed that the patients who received colchicine as the second-line therapy had a rate of response of $29 \% .^{13}$

This trial aims to study the efficacy of the combination therapy of colchicine and dapsone in cases of relapsed ITP, refractory ITP and steroid-dependent ITP. This combination may enhance the response rate due to different mechanisms of action. In addition, both these agents are inexpensive with different side effects.

\section{Materials and Methods}

This is a retrospective, chart-reviewed study on relapsed, refractory and steroiddependent ITP patients. This study was conducted from 1 January 2008 to 30 September 2014 at the Hematology Clinic, Chiang Mai University Hospital, Chiang Mai, Thailand. During that period, colchicine and dapsone combination thera- 
py was the first choice of second-line treatment in our center in cases of relapsed ITP, refractory ITP and steroid-dependent ITP, who did not have life threatening bleeding. The local Institutional Review Board approved the study stating it was in accordance with the guidelines in the declaration of Helsinki. The patients were included in the study if they were 18 years of age or older and had been diagnosed with one of following three types of ITP: 1

i: Relapsed ITP: the recurrence of platelet numbers less than $30 \times 10^{9} / \mathrm{L}$ in patients who had previously responded to at least 1 month of corticosteroid treatment.

ii: Refractory ITP: patients who were unresponsive to corticosteroids after 1 month of treatment with $1 \mathrm{mg} / \mathrm{kg} /$ day of prednisolone or equivalent dose of other corticosteroids.

iii: Steroid-dependent ITP: patients requiring at least $20 \mathrm{mg}$ /day of prednisolone to maintain a platelet count over $30 \times 10^{9} / \mathrm{L}$.

The patients were treated with a combination therapy of colchicine and dapsone with or without corticosteroids. Patients with a previous splenectomy or who had been previously treated with colchicine or dapsone monotherapy before that period were also included. Exclusion criteria were patients who received concurrent other second-line treatment and patients with G6PD deficiency.

\section{Treatment and follow-up}

Patients with relapsed, refractory or steroid dependent ITP were started on a treatment of prednisolone up to 1 $\mathrm{mg} / \mathrm{kg} /$ day. Colchicine (dose 0.6-1.2 $\mathrm{mg}$ /day) and dapsone (dose 50-100 mg/day) were started concurrently. Duration of treatment and dose adjustment for corticosteroid, colchicine and dapsone were decided to individual patient according to response. All the patients were followed-up by the assessment of the responsiveness of platelet counts, clinical bleeding events and treatment related complications.

\section{Data collection and treatment out- comes}

We collected the clinical data from electronic and paper medical records. The following information was retrieved: age, sex, co-morbidities, hemoglobin and platelet count at diagnosis, category of ITP (relapse, refractory, or steroid dependent), initial dose of prednisolone and discontinuation date, date of the start and finish of treatment with colchicine and dapsone, response, relapse following combination therapy, and adverse events.
The primary outcome of this study was the response rate of steroid-dependent, relapsed, and refractory ITP. The treatment responses were defined according to the following: ${ }^{1}$

i: Complete response (CR): a platelet count of $100 \times 10^{9} / \mathrm{L}$ or above for at least two consecutive measurements more than 7 days apart, without bleeding.

ii: Continuous complete response (CCR): patients who showed CR for at least 6 months.

iii: Response: a platelet count of $30 \times 10^{9} / \mathrm{L}$ or above that is at least double pretreatment levels for at least two consecutive measurements more than 7 days apart, without bleeding.

iv: No response (NR): a platelet count of fewer than $30 \times 10^{9} / \mathrm{L}$ or less that is less than double pretreatment levels for at least two consecutive measurements more than a day apart, or the presence of clinical bleeding.

The secondary outcomes were treatment related complications, bleeding events, all-causes mortality, and relapse rate.

\section{Statistical analyses}

Descriptive analysis was used to describe demographic data and clinical characteristics of the patients. Continuous data were reported using mean or median with corresponding standard deviation or range, as appropriate. Categorical data were reported using number with percentage. Time to response was defined by time from starting combination therapy to $\mathrm{CR}$ or response as defined previously. Time to corticosteroid discontinuation was defined by time from diagnosis of relapse, refractory, or steroid dependent to stopping prednisolone. Multivariate logistic regression was used to identify potential predictors for patient response to colchicine and dapsone combination therapy. Odds ratios (OR) with corresponding $95 \%$ confidence interval $(\mathrm{CI})$ were reported. Statistical significance was defined as a P-value less than 0.05. SPSS version 21.0 for Windows was used for all analysis.

\section{Results}

\section{Baseline characteristics}

Sixty-four ITP patients who received colchicine and dapsone combination therapy were included in this study. A median age was 46 years (range 15-73) with fortyfive patients $(70.3 \%)$ were female. Co-morbid diseases were reported in $37.5 \%$ of patients as shown in Table 1. All of them

Table 1. Demographic data of relapsed, refractory and steroid-dependent immune thrombocytopenia patients [total $(n=64)]$.

\begin{tabular}{lc} 
Patient characteristics & $\mathbf{N}(\%)$ \\
Gender & \\
Male & $19(29.7)$ \\
Female & $45(70.3)$ \\
Age at diagnosis, median (range) & $46(15-73)$ \\
\hline Co-morbid disease & \\
Yes & $24(37.5)$ \\
Endocrine (DM, DLP) & $10(15.6)$ \\
Cardiovascular (HT) & $9(14.1)$ \\
Respiratory (COPD) & $2(3.1)$ \\
Others & $3(4.7)$ \\
No & $40(62.5)$ \\
Category of ITP & \\
Relapsed & $42(65.6)$ \\
Refractory & $13(20.3)$ \\
Steroid-dependent & $9(14.1)$ \\
Platelet count at diagnosis, median (range)* & \\
Concomitant prednisolone & $8.44 \times 10^{9} / \mathrm{L}\left(3-69 \times 10^{9} / \mathrm{L}\right)$ \\
Yes & $60(93.7)$ \\
1 mg/kg/day & $36(56.2)$ \\
$<1$ mg/kg/day & $24(37.5)$ \\
No & $4(6.3)$ \\
Previous splenectomy & \\
Yes & $4(6.3)$ \\
No & $60(93.7)$ \\
\hline
\end{tabular}

*Missing platelet count data at diagnosis in one patient. DM, diabetes mellitus; DLP, dyslipidemia; HT, hypertension; COPD,chronic obstructive pulmonary disease. 
had primary ITP. Forty-two (65.6\%) were relapsed ITP patients, thirteen patients $(20.3 \%)$ were refractory ITP and nine patients $(14.1 \%)$ were steroid-dependent ITP. No patient in relapsed ITP group had previous episode of steroid-dependence.

The median platelet count before treatment was $22.6 \times 10^{9} / \mathrm{L}$ (range $3-69 \times 10^{9} / \mathrm{L}$ ). Forty-eight patients $(75 \%)$ had a platelet count $<30 \times 10^{9} / \mathrm{L}$. The most frequent prescribed colchicine dose was $1.2 \mathrm{mg} /$ day (58 patients, 90.6\%) and dapsone $100 \mathrm{mg} /$ day (52 patients, 96.3\%). Almost all except 4 patients $(93.7 \%)$ received concomitant prednisolone. Of these, 36 patients $(56.2 \%)$ were treated with a prednisolone dose of 1 $\mathrm{mg} / \mathrm{kg} /$ day at the time of second line treatment. The remaining patients received prednisolone at lower doses (range 10-40 $\mathrm{mg}$ /day). Only four (6.3\%) patients had splenectomy before second-line management. (Table 1) No patients who had previous exposure to either colchicine or dapsone were included in the study.

\section{Response to treatment}

Fifty-three patients $(82.8 \%)$ responded to the combination therapy, with 48 patients (75.0\%) achieved CR whereas eleven patients $(17.2 \%)$ had NR. (Table 2)

In each indication of second-line therapy, overall response rate was higher in relapsed ITP patients at $90.4 \%$ compared to refractory $(61.5 \%)$ and steroid-dependent ITP cases $(77.8 \%)$. Refractory ITP resulted in the highest NR [5 patients $(38.5 \%)$ ] (Table 2).

The median time to response was 8 weeks (interquartile range 26-44). Of the 60 patients who received concomitant prednisolone, 30 patients $(50 \%)$ were able to discontinue prednisolone, with a median time of termination of prednisolone of 39.9 weeks. The remaining patients who received prednisolone were able to decrease the dosage to a median of $5 \mathrm{mg} /$ day.

In the group of ITP patients who responded to treatment, fifteen patients (28.3\%) had CCR $(18.8 \%, 5.7 \%$, and $3.8 \%$ in relapsed ITP, steroid dependent ITP and refractory ITP, respectively) at the median follow up 22.5 months. Five patients $(9.4 \%)$ were able to discontinue both colchicine and dapsone, while remaining at CCR. However, twenty three $(43.4 \%)$ patients eventually relapsed $(39.6 \%$ of the relapsed ITP group and $3.8 \%$ of the steroid-dependent ITP group) with a median time from response to recurrence of 64.2 weeks (range 7-172 weeks). The majority of them (14 patients, $60.9 \%$ ) received both colchicine and dapsone at the time of relapsed whereas 2 patients $(8.7 \%)$ already discontinued both drugs and 7 patients $(30.4 \%)$ had either colchicine or dapsone when relapsed.

Thirty four patients $(53.1 \%)$ continued both dapsone and colchicine combination therapy until the last follow-up. In the contrary, $14(21.9 \%)$ patients discontinued both medications with 4 patients stopped simultaneously and 6 patients stopped colchicine before dapsone. Sixteen (25\%) patients quit only dapsone or colchicine ( 8 patients in each group). The majority of patients (23 from 30 patients, $76.7 \%$ ) stopped medications without tapering of doses. The median duration of treatment with dapsone and colchicine were comparable at 14.5 months (range 1-63 and 1-61 months, respectively).

\section{Adverse events}

Ten patients $(15.6 \%)$ experienced adverse events, of which eight patients $(12.5 \%)$ had dapsone-related hemolysis defined by anemia with laboratory evidences of hemolysis (5 with subclinical hemolysis with hemoglobin more than 10 $\mathrm{g} / \mathrm{dL})$. The other 2 patients (3.1\%) had transaminase enzyme elevation with peak values of 2.7 times of upper normal range. One patient with hepatitis discontinued colchicine and subsequently responded to dapsone monotherapy. Nevertheless, he still had persistent mild transaminitis and nonalcoholic steatohepatitis was diagnosed later. Another patient had transient elevation of liver enzymes and not required cessation of medication. No patient suffered from infectious complications. However, 10 patients $(15.6 \%)$ died of unknown reasons as they were lost to follow up.

\section{Clinical factors that affected the treatment response}

Relapsed ITP group was significantly correlate with treatment response compare to composite refractory ITP and steroiddependent ITP group [OR 4.43 (95\%CI: $0.93-23.22, \mathrm{P}=0.037]$. There were no statistical differences between sex, age, and platelet count at initial diagnosis of ITP in the treatment response. Regarding the other treatment effects, there were no differences between concomitant prednisolone of 1

Table 2. Demographic data and treatment response according to category of immune thrombocytopenia.

\begin{tabular}{|c|c|c|c|c|}
\hline & Overall ITP $n=64$ & Relapsed $n=42$ & $\begin{array}{l}\text { Category of ITP } \\
\text { Steroid-dependent } n=9\end{array}$ & Refractory $\mathrm{n}=13$ \\
\hline Median age in years (age range) & $46(15-73)$ & $46(15-73)$ & $50(16-68)$ & $42(18-59)$ \\
\hline Mean platelet count at diagnosis $\left(\times 10^{9} / \mathrm{L}\right)$ & 22.6 & 20.1 & 29.2 & 17.3 \\
\hline Previous splenectomy, n (\%) & $4(6.2)$ & $2(50.0)$ & $0(0.0)$ & $2(50.0)$ \\
\hline Concomitant prednisolone $1 \mathrm{mg} / \mathrm{kg} /$ day, n (\%) & $36(56.2)$ & $26(72.2)$ & $1(2.8)$ & $9(25.0)$ \\
\hline Response, n (\%) & $53(82.8)$ & 38 (71.7) & $7(13.2)$ & $8(15.1)$ \\
\hline Complete response, $\mathrm{n}(\%)$ & $48(75.0)$ & $35(72.9)$ & $7(14.6)$ & $6(12.5)$ \\
\hline Response but not CR, n (\%) & $5(7.8)$ & $3(60.0)$ & $0(0)$ & $2(40.0)$ \\
\hline No response, $\mathrm{n}(\%)$ & $11(17.2)$ & $4(36.4)$ & $2(18.2)$ & $5(45.4)$ \\
\hline Median time to response (weeks) & 8 & 8 & 8 & 6 \\
\hline Prednisolone discontinuation, $\mathrm{n}(\%)^{*}$ & $30(50)$ & $23(76.7)$ & $4(13.3)$ & $3(10.0)$ \\
\hline Adverse effects, n (\%) & $10(15.6)$ & $7(70.0)$ & $1(10.0)$ & $2(20.0)$ \\
\hline $\begin{array}{l}\text { Outcome, } n(\%) \\
\text { CCR** } \\
\text { Relapsed after response** } \\
\text { Died } \\
\text { Loss of follow up }\end{array}$ & $\begin{array}{l}15(28.3) \\
23(43.4) \\
10(15.6) \\
11(17.2)\end{array}$ & $\begin{array}{c}10(66.7) \\
21(91.3) \\
6(60.0) \\
2(18.2)\end{array}$ & $\begin{array}{l}3(20.0) \\
2(8.7) \\
1(10.0) \\
2(18.2)\end{array}$ & $\begin{array}{c}2(13.3) \\
0(0) \\
3(30.0) \\
7(63.6)\end{array}$ \\
\hline
\end{tabular}

*Sixty patients received concomitant prednisolone. ${ }^{* *}$ Compared to 53 patients who showed overall response, including 10 cases lost to follow up. CR, complete response; R, response; CCR, continuous complete response. 
$\mathrm{mg} / \mathrm{kg} /$ day or a lower dosage and also between patients who had undergone splenectomy versus intact-spleen individuals (Table 3 ).

\section{Discussion}

We reported a high response rate from a combination of colchicine and dapsone in concomitant with steroid especially in relapsed, ITP patients. Refractory and steroid dependent ITP patients had lower response rate compared to relapse group. The median time of response was 8 weeks. Half patients were able to discontinue prednisolone. Fifteen patients $(28.3 \%)$ had CCR with these combination treatments. Furthermore, this combination treatment had minimal side effects and was non-invasive compared to other second-line modalities for ITP treatment.

A systematic review of the use of splenectomy for treatment of ITP showed a response rate of $88 \%$ and a $\mathrm{CR}$ rate of $66 \% .{ }^{8}$ Moreover, splenectomy had a higher rate of sustained response with a median relapse rate of $15 \%{ }^{8}$ This is compared to only one-third of patients in this study who maintained CCR. The limitations of a splenectomy are mainly due to its complications. Complication rates were $12.9 \%$ with a laparotomy and $9.6 \%$ with a laparoscopic splenectomy and mortality rates were $1 \%$ and $0.2 \%$, respectively. ${ }^{8}$ Since there are no preoperative characteristics to help predict patient response to a splenectomy, ${ }^{8}$ which may lead to bleeding complications, many medical treatments have been investigated to facilitate second-line treatment of ITP enabling a splenectomy to be deferred until the chronic phase of disease (12 months). ${ }^{2,14}$ In one large cohort study, carried out over the last 35 years, use of a splenectomy tended to delay second- to third-line therapy but the overall rate of operation remained steady at around 15$25 \% .{ }^{15}$ The emerging therapies as a secondline treatment were rituximab and thrombopoietin receptor agonists. ${ }^{15}$

Rituximab has been studied for treatment of ITP. An early systematic review derived from uncontrolled studies showed an overall platelet count response in $62.5 \%{ }^{16}$ that was similar to the current study. The international guidelines recommend that rituximab may be considered for patients where one line of therapy has failed, ${ }^{2}$ even though its use is limited by cost and toxicity and a mortality rate of $2.9 \%{ }^{16}$ Moreover, the later RCT for second-line treatment could not show significant benefit of rituximab over placebo in terms of long-term treatment failure or overall response rate. ${ }^{17}$ When comparing the results of the colchicine and dapsone combination therapy with the placebo group in that trial, the combination therapy seems to improve overall response rate $(82.8 \% \mathrm{vs}$ $67 \%)$, CR rate $(72.2 \%$ vs $39 \%$ ) along with a decreased relapse rate $(43.4 \%$ vs $68 \%$, respectively) ${ }^{16}$

Thrombopoietin receptor agonists are novel treatments that have been investigated in this field. Eltrombopag is an oral ago- nist that has been used in a phase 3 RCT in previous treated ITP patients who had a platelet count of less than $30 \times 10^{9} / \mathrm{L}$ for more than 6 months. The results were impressive since eltrombopag had a better response rate $(79 \%$ vs $28 \%)$ compare to placebo. ${ }^{17}$ The response rate of patients on eltrombopag is slightly higher than a subgroup of refractory ITP patients $(61 \%)$ and comparable with steroid-dependent patients (77.8\%), who received combination therapy in the current study. While a long-term study supported a durable response of patients on ethrombopag, $13 \%$ of patients experienced adverse events that led to withdrawal. These adverse effects included in particular, thromboembolic events and liver enzyme elevation. ${ }^{19}$ Another thrombopoietin receptor agonist that has been evaluated in RCT is romiplostim. The overall response rate was $88 \%$ and $79 \%$ of nonsplenectomised and splenectomised patients respectively, ${ }^{20}$ which were comparable to eltrombopag but little higher than the results in the current study. A study of longterm safety and tolerability of romiplostim revealed some of the same adverse events as eltrombopag. ${ }^{21}$

Focusing now on chemotherapy and immunosuppressive therapy, the use of vincristine has been studied in both newly diagnosed and refractory ITP patients. ${ }^{22,23}$ Vincristine showed a high response rate of $75.8 \%$ with a median time of response of 9 days in relapsed or refractory patients. ${ }^{23}$ This data support that vincristine provides similar efficacy to colchicine and dapsone combination but the advantage is that it has

Table 3. Correlation of risk factors and treatment response.

\begin{tabular}{|c|c|c|c|c|c|}
\hline Variables & $1(n=64)$ & Response (n=53), n (\%) & & ariate ana & \\
\hline & & & OR & $95 \%$ CI & P-value \\
\hline Gender & & & 2.32 & $0.47-10.66$ & 0.279 \\
\hline Female & 45 & $39(73.6)$ & & & \\
\hline Male & 19 & $14(26.4)$ & & & \\
\hline Age at diagnosis & & & 0.72 & $0.11-3.55$ & 1.000 \\
\hline$\geq 40$ years & 43 & $35(66.0)$ & & & \\
\hline$<40$ years & 21 & $18(34.0)$ & & & \\
\hline ITP category & & & 4.43 & $0.93-23.22$ & 0.037 \\
\hline Relapsed & 42 & 38 (71.7) & & & \\
\hline Refractory and steroid-dependent & 22 & $15(28.3)$ & & & \\
\hline Platelet at diagnosis & & & 0.24 & $0.00-2.05$ & 0.263 \\
\hline$\geq 15 \times 10^{9} / \mathrm{L}$ & 47 & $37(71.2)$ & & & \\
\hline$<15 \times 10^{9} / \mathrm{L}$ & 16 & $15(28.8)$ & & & \\
\hline Prednisolone concomitant & & & 2.32 & $0.10-3.83$ & 0.729 \\
\hline $1 \mathrm{mg} / \mathrm{kg} /$ day & 36 & $30(58.8)$ & & & \\
\hline <1mg/kg/day & 24 & $21(41.2)$ & & & \\
\hline Previous splenectomy & & & 0.60 & $0.04-34.60$ & 0.539 \\
\hline Splenectomy & 4 & $3(5.7)$ & & & \\
\hline Non-splenectomy & 60 & $50(94.3)$ & & & \\
\hline
\end{tabular}

OR, odds ratio; CI, confidence interval. 
shorter time to response. However, the sustained response is around $20 \%$ and peripheral neuropathy is a common adverse event. 22,23

The using of immunosuppressive therapy in ITP has declined over time. ${ }^{15}$ These agents include cyclophosphamide, azathioprine, cyclosporine, and mycophenolate mofetil. Many case studies have shown a varied overall response rate of approximately $40-83.3 \%$ in refractory ITP. ${ }^{2,24-27}$ Since there have been no large prospective clinical trials about immunosuppression in ITP, the international guidelines cannot provide an appropriate indication or timing of this kind of treatment in ITP. ${ }^{2}$

The combination therapy of colchicine and dapsone for second-line treatment of ITP is an interesting option because it is much cheaper than rituximab or eltrombopag and does not have the serious side effects associated with immunosuppressive therapy. It seems that the combination therapy led to a higher response rate when compared to dapsone monotherapy and colchicine monotherapy. ${ }^{9-13}$ However, the median time of response that occurred within 8 weeks is not significantly different from monotherapy (3-14 weeks for dapsone and 2 weeks for colchicine)

A previous study of a combination of dapsone and colchicine in 10 patients with refractory and steroid-dependent ITP showed $40 \%$ CR and CCR of $20 \% .^{29}$ These efficacy results are slightly lower than the current study which showed a CR rate of $77.8 \%$ and $46.1 \%$ in steroid dependent and refractory cases, respectively and CCR of $28.3 \%$. It might be explained by the lower median dose of prednisolone of $20 \mathrm{mg}$ /day in the previous study. ${ }^{28}$

Another drug that is useful in this setting is danazol which is an attenuated androgen..$^{5}$ The reported response rate was $67 \%$ with $46 \%$ of patients exhibiting durable remission, acceptable tolerability. ${ }^{29}$ As a result, the combination therapy of danazol, colchicine, and dapsone is an attractive option for a future clinical trial.

Since the patient characteristics, inclusion criteria and response criteria were different between the trials. As a result, it was difficult to directly compare the efficacy of different therapies to our current study. Our study had more relapsed ITP patients which trended to response to treatment more than relapsed group. In addition, more than $90 \%$ of patients received concomitant prednisolone with more than half being given a dose of $1 \mathrm{mg} / \mathrm{kg} / \mathrm{day}$, the author could not determine the causality or magnitude of the effect - whether it was derived from the colchicine and dapsone combination therapy, concomitant prednisolone, or both.
Especially in relapsed patients who previously responded to prednisolone treatment as a front-line therapy.

In spite of the high response rate in our study, this also can be explained from the unique population. The median platelet count before starting treatment was $22.6 \times 10^{9} / \mathrm{L}$ which was not very low. The median time to response was long as 2 months. So, this combination treatment may not suitable for the severe patients who had life threatening bleeding and require rapid response from second line treatment.

On the other hand, half the patients receiving the combined therapy were able to discontinue steroids, $9.4 \%$ were able to discontinue both colchicine and dapsone and $28.3 \%$ experienced CCR. These results may represent the benefit of this combination treatment. Limitations of this study included its retrospective methodology and small sample size. As a nature in this type of study, selection bias of patients, missing data especially side effect and adverse event were the crucial limitation. Further studies should be prospective in design and include a larger population to confirm the efficacy of the colchicine and dapsone combination therapy.

\section{Conclusions}

In summary, this study showed an alternative second-line therapy option of a combination of colchicine and dapsone in relapsed ITP cases with acceptable side effects. These agents offer potential as a cost-effective treatment of second-line ITP.

\section{References}

1. Provan D, Stasi R, Newland AC, et al. International consensus report on the investigation and management of primary immune thrombocytopenia. Blood 2010;115:168-86.

2. Neunert C, Lim W, Crowther M, et al. The American Society of Hematology 2011 evidence-based practice guideline for immune thrombocytopenia. Blood 2011;117:4190-207.

3. Frederiksen H, Schmidt K. The incidence of idiopathic thrombocytopenic purpura in adults increases with age. Blood 1999;94:909-13.

4. Terrell DR, Beebe LA, Vesely SK, et al. The incidence of immune thrombocytopenic purpura in children and adults: A critical review of published reports. Am J Hematol 2010;85:174-80.

5. British Committee for Standards in
Haematology General Haematology Task Force. Guidelines for the investigation and management of idiopathic thrombocytopenic purpura in adults, children and in pregnancy. $\mathrm{Br} \mathrm{J}$ Haematol 2003;120:574-96.

6. den Ottolander GJ, Gratama JW, de Koning J, Brand A. Long-term followup study of 168 patients with immune thrombocytopenia. Implications for therapy. Scand J Haematol 1984; $32: 101-10$

7. DiFino SM, Lachant NA, Kirshner JJ, Gottlieb AJ. Adult idiopathic thrombocytopenic purpura. Clinical findings and response to therapy. Am J Med 1980;69:430-42.

8. Kojouri K, Vesely SK, Terrell DR, George JN. Splenectomy for adult patients with idiopathic thrombocytopenic purpura: a systematic review to assess long-term platelet count responses, prediction of response, and surgical complications. Blood 2004;104:262334.

9. Vancine-Califani SM, De Paula EV, Ozelo MC, et al. Efficacy and safety of dapsone as a second-line treatment in non-splenectomized adults with immune thrombocytopenic purpura. Platelets 2008;19:489-95.

10. Godeau B, Durand JM, RoudotThoraval F, et al. Dapsone for chronic autoimmune thrombocytopenic purpura: a report of 66 cases. Br J Haematol 1997;97:336-9.

11. Hernandez F, Linares M, Colomina P, et al. Dapsone for refractory chronic idiopathic thrombocytopenic purpura. Br J Haematol 1995;90:473-5.

12. Damodar S, Viswabandya A, George B, et al. Dapsone for chronic idiopathic thrombocytopenic purpura in children and adults: a report on 90 patients. Eur J Haematol 2005;75:328-31.

13. Strother SV, Zuckerman KS, LoBuglio AF. Colchicine therapy for refractory idiopathic thrombocytopenic purpura. Arch Intern Med 1984;144:2198-200.

14. Mahevas M, Michel M, Godeau B. How we manage immune thrombocytopenia in the elderly. $\mathrm{Br} \mathrm{J}$ Haematol 2016;173:844-56.

15 Palandri F, Polverelli N, Sollazzo D, et al. Have splenectomy rate and main outcomes of ITP changed after the introduction of new treatments? A monocentric study in the outpatient setting during 35 years. Am J Hematol 2016;91:e267-72.

16. Arnold DM, Dentali F, Crowther MA, et al. Systematic review: efficacy and safety of rituximab for adults with idiopathic thrombocytopenic purpura. Ann 
Intern Med 2007;146:25-33.

17. Ghanima W, Khelif A, Waage A, et al. Rituximab as second-line treatment for adult immune thrombocytopenia (the RITP trial): a multicentre, randomised, double-blind, placebo-controlled trial. Lancet 2015;385:1653-61.

18. Cheng G, Saleh MN, Marcher C, et al. Eltrombopag for management of chronic immune thrombocytopenia (RAISE): a 6-month, randomised, phase 3 study. Lancet 2011;377:393-402.

19 Saleh MN, Bussel JB, Cheng G, et al. Safety and efficacy of eltrombopag for treatment of chronic immune thrombocytopenia: results of the long-term, open-label EXTEND study. Blood 2013;121:537-45.

20. Kuter DJ, Bussel JB, Lyons RM, et al. Efficacy of romiplostim in patients with chronic immune thrombocytopenic purpura: a double-blind randomised controlled trial. Lancet 2008;371:395-403.

21. Rodeghiero F, Stasi R, Giagounidis A, et al. Long-term safety and tolerability of romiplostim in patients with primary immune thrombocytopenia: a pooled analysis of 13 clinical trials. Eur J Haematol 2016;91:423-36.

22. Stirnemann J, Kaddouri N, Khellaf M, et al. Vincristine efficacy and safety in treating immune thrombocytopenia: a retrospective study of 35 patients. Eur J Haematol 2016;96:269-75.

23. Park YH, Yi HG, Lee ML, et al. Clinical efficacy and tolerability of vincristine in splenectomized patients with refractory or relapsed immune thrombocytopenia: a retrospective single-center study. Int J Hematol 2016;103:180-8.

24. Reiner A, Gernsheimer T, Slichter SJ. Pulse cyclophosphamide therapy for refractory autoimmune thrombocytopenic purpura. Blood 1995;85:351-8.

25. Quiquandon I, Fenaux P, Caulier MT, et al. Re-evaluation of the role of azathioprine in the treatment of adult chronic idiopathic thrombocytopenic purpura: a report on 53 cases. $\mathrm{Br} \mathrm{J}$ Haematol 1990;74:223-8.

26. Emilia G, Morselli M, Luppi M, et al. Long-term salvage therapy with cyclosporin A in refractory idiopathic thrombocytopenic purpura. Blood 2002;99:1482-5.

27. Provan D, Moss AJ, Newland AC, Bussel JB. Efficacy of mycophenolate mofetil as single-agent therapy for refractory immune thrombocytopenic purpura. Am J Hematol 2006;81:19-25.

28. Uoiyeunporn M, Rojnuckarin P. Efficacy of dapsone and colchicine for immune thrombocytopenia (ITP): 6year experience in King Chulalongkorn Memorial Hospital. J Hematol Transfus Med 2010;20:119-26.

29. Maloisel F, Andrès E, Zimmer J, et al. Danazol therapy in patients with chronic idiopathic thrombocytopenic purpura: long-term results. Am J Med 2004;116:590-4. 